\title{
AVALIAÇÃO DE ALGORITMOS DE CLASSIFICAÇÃO SUPERVISIONADA DE IMAGEM DIGITAL DO SATÉLITE IKONOS NA REGIÃO DA SERRA DO SALITRE - MG
}

\author{
Julierme Wagner da Penha \\ Universidade Federal de Viçosa - UFV \\ juliermewagner@yahoo.com.br
}

\section{RESUMO}

Diversas técnicas de processamento de imagens digitais permitem tratar os dados e gerar parâmetros adicionais aos métodos convencionais de pesquisa. Dentre estas técnicas, temos a classificação supervisionada, que atribui a cada pixel da imagem uma dada classe, a fim de reconhecer padrões e objetos para mapear áreas de interesse. Cada pixel apresenta um nível de cinza (valor numérico), ou seja, representa a reflectância dos objetos que o compõem. Como produto final tem-se um mapa temático, o qual representa a distribuição espacial das classes temáticas. A classificação apresenta-se de dois tipos: supervisionada e não supervisionada. A classificação supervisionada depende de amostras de treinamento que sejam representativas das classes presentes na imagem. Assim, o algoritmo classifica os pixels para cada classe. As amostras de treinamento devem ser em número representativo e uniforme. Enquanto que a classificação não supervisionada dispensa a definição do número de classes e das amostras de treinamento, uma vez que o algoritmo agrega pixels e o intérprete identifica as classes geradas pelo algoritmo. O objetivo deste trabalho foi descrever as etapas empregadas no procedimento de classificação e avaliar dois algoritmos de classificação supervisionada, o da Distância Mínima até a Média e o da Máxima Verossimilhança utilizando uma imagem de satélite IKONOS da região da Serra do Salitre, no Triângulo Mineiro, no estado de Minas Gerais, a fim de verificar qual fornece a melhor classificação e conseqüente mapa temático.

\section{ABSTRACT}

Several techniques of processing digital images to process data and generate additional parameters to the conventional methods of research. Among these techniques, we have the classification, since each pixel of the image attached to a given class in order to recognize patterns and objects to map areas of interest. Each pixel displays a gray-level (numeric value), or represents the reflectance of the objects which compose it. As the final product has a thematic map, this represents the spatial distribution of thematic classes. The classification is presented in two types: supervised and not supervised. The supervised classification depends on the training samples that are representative of the classes in the image. Thus, the algorithm classifies the pixels for each class. The training samples must be representative and uniform in number. While the classification does not relieve the supervised setting of the number of classes and training samples, since the algorithm adds pixels and the interpreter identifies the classes generated by the algorithm. The objective of this study was to describe the steps used in the procedure of classification and evaluate two algorithms for supervised classification, the minimum distance to the average and the maximum likelihood using an IKONOS satellite image of the region of Serra do Salitre, the Triangle Mineiro in state of Minas Gerais, to see which provides the best classification and resulting thematic map. 


\section{INTRODUÇÃO}

Classificação de imagens pode ser entendida como o processo de extração de informações de modo a permitir o reconhecimento de padrões e objetos homogêneos com intuito de mapear as áreas da superfície terrestre (Fonseca, 2000). Este processo pode ter duas etapas, a primeira delas relativa ao reconhecimento de categorias de objetos do mundo real e a segunda na identificação de entidades a serem classificadas (Mather, 1999).

Os tipos de classificação podem ser: supervisionada e não supervisionada. $\mathrm{Na}$ classificação supervisionada, faz-se necessário que o usuário conheça algo relativo à área que será classificada antes de iniciar o processo. A classificação não supervisionada fundamenta-se no princípio que o software seja capaz de identificar por si só as classes inseridas num conjunto de dados (Crósta, 1993).

Diversos são os algoritmos disponíveis para classificação supervisionada de imagens, entre os quais merecem destaque: o da distância Mínima até a Média e o da Máxima Verossimilhança.

O algoritmo da Distância Mínima até a Média utiliza a média das amostras de treinamento para assinalar um pixel desconhecido a uma dada classe, atribuindo-o à classe cuja média é a mais próxima (Soares, 2000; Crósta, 1993).

O algoritmo da Máxima Verossimilhança utiliza a média e a covariância das amostras de treinamento, computando a probabilidade estatística de um pixel desconhecido pertencer a uma ou outra classe através de funções de densidade de probabilidade. Após avaliar a probabilidade para cada categoria, o pixel é assinalado à classe cuja probabilidade tenha sido a mais alta, ou mesmo a nenhuma delas para o caso em que os valores de probabilidade estiverem abaixo de um certo limiar estipulado pelo usuário (Soares, 2000).

Após a classificação de uma imagem de sensoriamento remoto é necessário avaliar a qualidade do produto obtido. Para isso, utiliza-se um segundo mapa denominado imagem de referência, cujas classes e os tamanhos dos pixels sejam similares aos da imagem classificada (Soares, 2000); ou então, pode-se utilizar uma imagem de referência mediante a localização e amostragem aleatória de pixels.

O objetivo deste trabalho é descrever as etapas empregadas no procedimento de classificação de dois algoritmos, o da Distância Mínima até a Média e o da Máxima Verossimilhança (MAXVER) utilizando uma imagem de satélite IKONOS da região da Serra do Salitre, no Triângulo Mineiro, no estado de Minas Gerais, com avaliação dos resultados obtidos com a classificação para cada um dos algoritmos, a posteriori.

\section{MATERIAL E MÉTODOS}

Foi utilizada uma imagem de satélite IKONOS da região da Serra do Salitre, no Triângulo Mineiro, no estado de Minas Gerais.

O software empregado no procedimento foi o Erdas Imagine versão 8.5. Prioritariamente realizou-se a seleção das amostras de treinamento com base em sete classes informacionais, a saber: campo limpo, campo sujo, café, braquiária, mata ciliar, pasto e solo. O número de amostras de treinamento por classe foi estabelecido considerando o critério de que este número deveria ser de cinco a dez, sendo que para este trabalho utilizou-se o número de sete amostras de treinamento por classe.

Determinou-se que todas as amostras de treinamento deveriam apresentar o mesmo tamanho assim como o mesmo número de pixels por classe, sendo considerado o critério de que esse número deveria estar no intervalo de cem a quinhentos. Para isso, considerou-se dezesseis (16) pixels (polígono quadrado) por amostra e sete (7) amostras por classe de forma que cada classe contou com cento e doze (112) pixels amostrais. 
A etapa seguinte à seleção de amostras de treinamento consistiu da avaliação de sua qualidade, utilizando elementos como a matriz de contingência (entre as sete amostras e as respectivas classes), o histograma (entre as sete amostras para as quatro bandas) e a divergência transformada (entre as quatro bandas para as quarenta e nove amostras). Após a avaliação das amostras, procedeu-se com sua edição, realizando operação de fusão das mesmas.

Considerando-se as amostras de treinamento devidamente avaliadas e editadas, procedeu-se com a classificação propriamente dita, empregando para isso os algoritmos da Distância Mínima até a Média e da Máxima Verossimilhança.

Finalmente, submeteu-se as imagens classificadas a uma avaliação da exatidão por meio de uma localização aleatória de duzentos e cinqüenta (250) pixels, baseada na imagem classificada, constituindo assim uma imagem de referencia para comparação de cada um dos algoritmos utilizados, através dos parâmetros: índice de exatidão total (global), índice kappa geral e índice kappa condicional.

\section{RESULTADOS E DISCUSSÃO}

Os resultados da avaliação das amostras de treinamento quanto à matriz de contingência mostraram que houve pouca confusão. A maior confusão observada foi entre as amostras referentes às classes de mata ciliar e café, entretanto, não houve necessidade de eliminá-la, pois se encontrava em padrões aceitáveis.

A avaliação das amostras de treinamento pelo histograma também mostra distribuição dos valores numéricos entre as classes e para cada banda espectral, conforme podem ser observado na ilustração das Figuras $1,2,3,4,5,6$ e 7 .

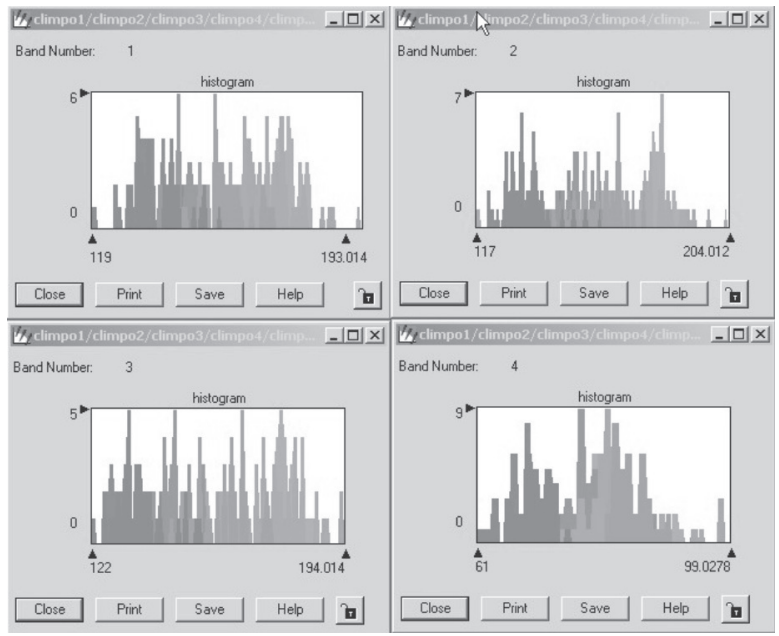

Figura 1. Histogramas de avaliação da classe campo limpo.

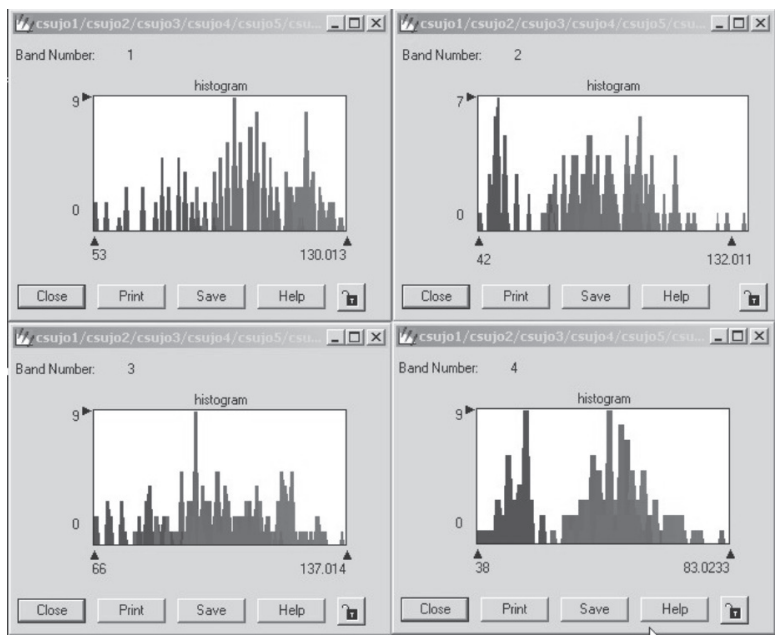

Figura 2. Histogramas de avaliação da classe campo sujo.

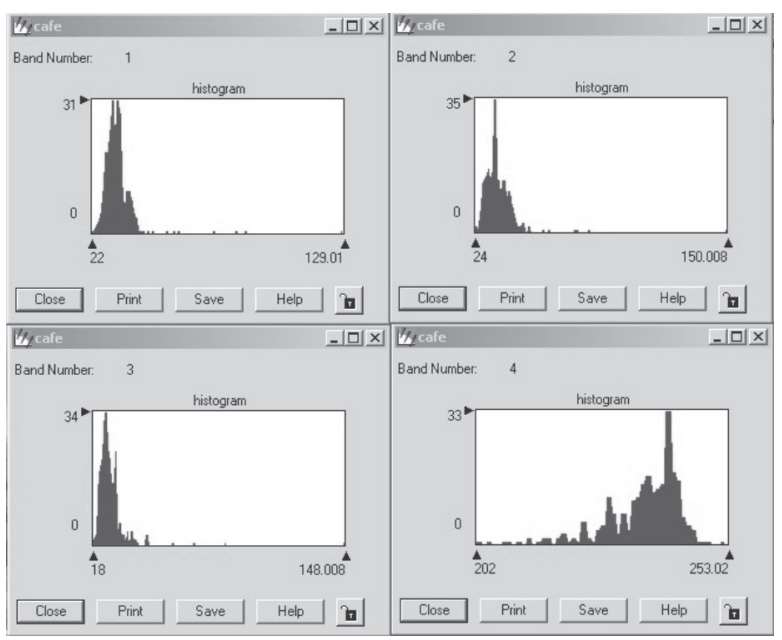

Figura 3. Histogramas de avaliação da classe café. 


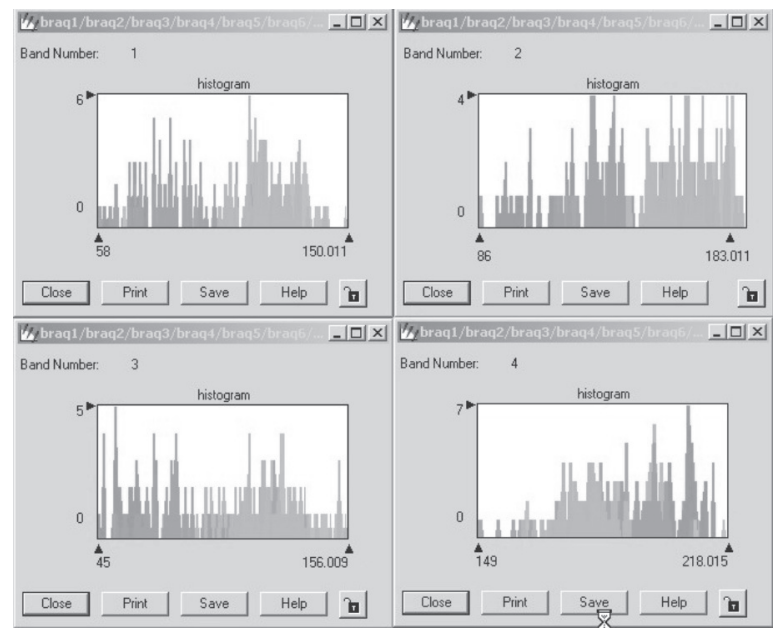

Figura 4. Histogramas de avaliação da classe braquiária.

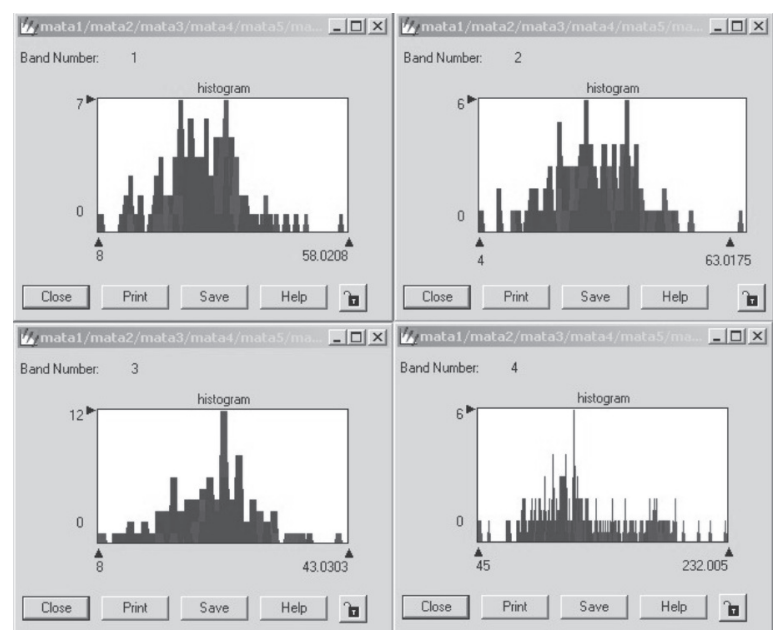

Figura 5. Histogramas de avaliação da classe mata ciliar.

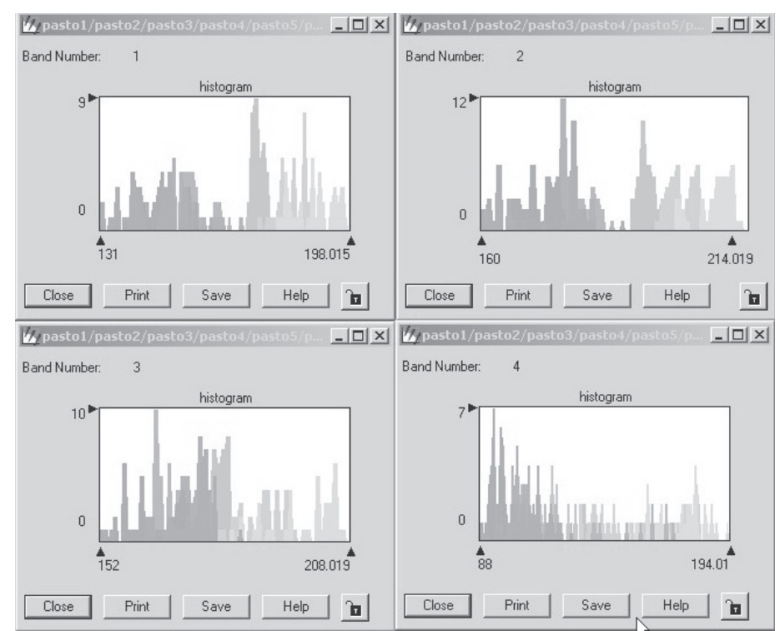

Figura 6. Histogramas de avaliação da classe pasto.

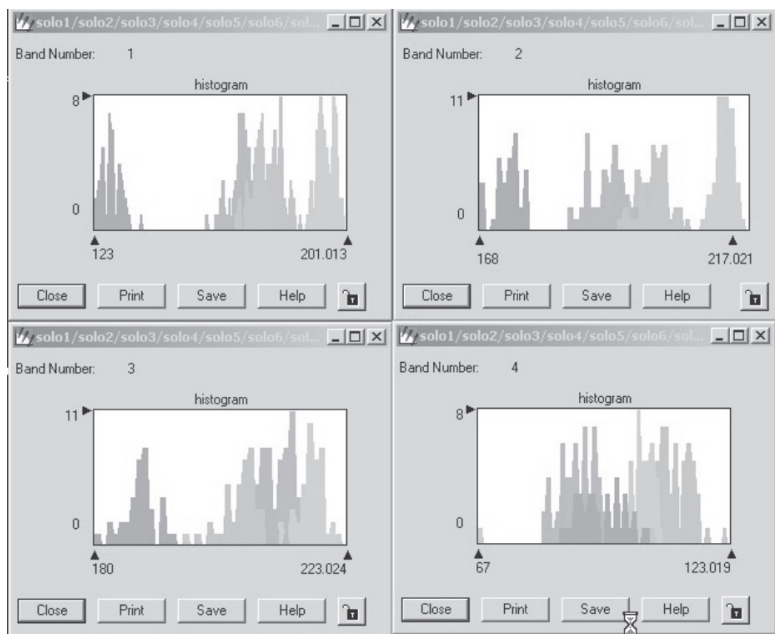

Figura 7. Histogramas de avaliação da classe solo.

É sabido que os histogramas dessas classes nas respectivas bandas espectrais da imagem fornecem informações úteis para se fazer várias análises. Pois, esses histogramas revelam a distribuição dos níveis de cinza desta imagem em cada classe, ele é representado por um gráfico que dá o número de pixels na imagem para cada nível de cinza dentro da sua classe.

Ao analisar a qualidade das amostras de treinamento relacionadas quanto a sua divergência, através da divergência transformada, constatou-se que as médias observadas para as quatro bandas foram acima de um mil e novecentos (1900), podendo ser classificadas como excelente.

Os produtos obtidos com a classificação pelo algoritmo da Distância Mínima até a Média (Figura 8 (a)) e pelo algoritmo da Máxima Verossimilhança (Figura 8 (b)) apresentaram poucas diferenças visuais entre si.

Entretanto, o método da Máxima Verossimilhança apresentou melhores resultados na avaliação da qualidade do produto temático final conforme pode ser observado pelos parâmetros de avaliação Producer's Accuracy, User's Accuracy e Kappa Condicional na Tabela 1. 


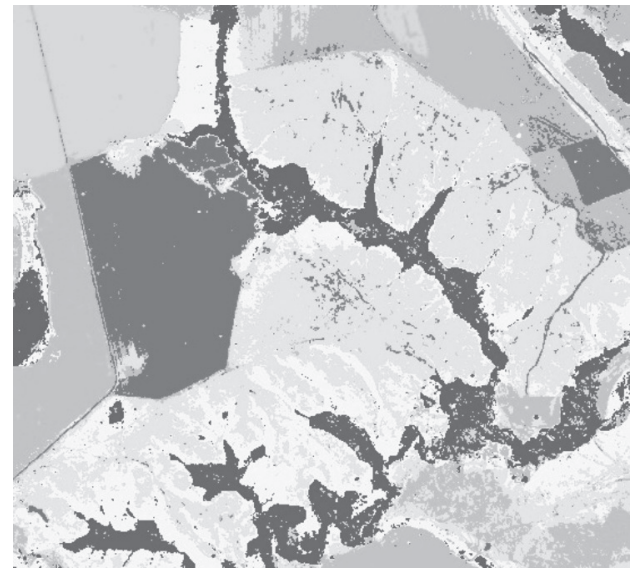

(a)

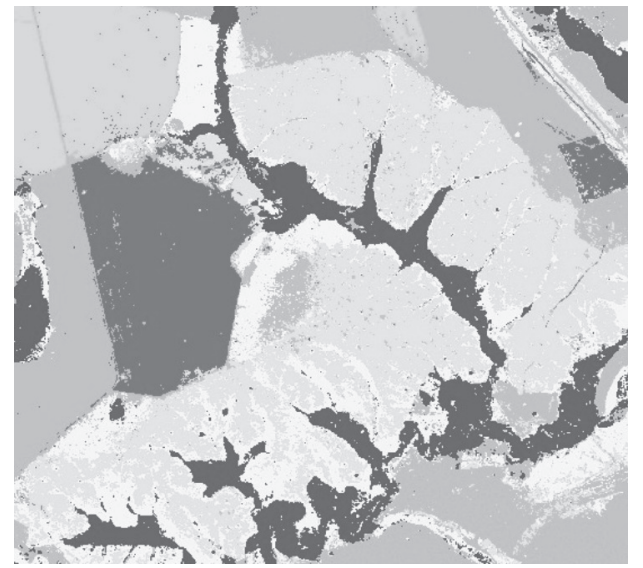

(b)

Figura 8. Imagem classificada obtida pelo algoritmo da Distância Mínima até a Média (a); Imagem classificada obtida pelo algoritmo da Máxima Verossimilhança (b).

Tabela 1. Resultados dos parâmetros de avaliação Producer's Accuracy, User's Accuracy e Kappa Condicional por classe e por algoritmo utilizado, onde DM: Distância Mínima até a Média; MV: Máxima Verossimilhança.

\begin{tabular}{lcrrrrr}
\hline \multicolumn{1}{c}{ Classes } & \multicolumn{2}{c}{ Producer's Accuracy } & \multicolumn{2}{c}{ User's Accuracy } & \multicolumn{2}{c}{ Kappa condicional } \\
& \multicolumn{1}{c}{ DM } & \multicolumn{1}{c}{ MV } & \multicolumn{1}{c}{ DM } & \multicolumn{1}{c}{ MV } & \multicolumn{1}{c}{ DM } & MV \\
\hline Campo limpo & 59,38 & 80,67 & 79,17 & 89,36 & 0,7200 & 0,8657 \\
Campo sujo & 100,00 & 90,73 & 10,26 & 74,36 & 0,0880 & 0,7060 \\
Café & 100,00 & 100,00 & 100,00 & 100,00 & 1,0000 & 1,0000 \\
Braquiária & 95,65 & 92,86 & 73,33 & 81,25 & 0,7063 & 0,7889 \\
Mata ciliar & 65,38 & 89,47 & 100,00 & 100,00 & 1,0000 & 1,0000 \\
Pasto & 64,94 & 84,38 & 85,29 & 75,00 & 0,8207 & 0,7133 \\
Solo & 90,32 & 81,58 & 82,35 & 96,88 & 0,7985 & 0,9631 \\
\hline
\end{tabular}

Constatou-se a superioridade do algoritmo da Máxima Verossimilhança (MV) em relação ao da Distância Mínima até a Média (DM) pelos parâmetros de avaliação da classificação geral como Índice de Exatidão Total (IET) e Índice Kappa Geral (IKG). Foram verificados os valores de IET e IKG de: 74,40 e 0,6996 respectivamente, para o método DM, e, IET e IKG de: 87,60 e 0,8547 respectivamente para o método $\mathrm{MV}$.
Para ilustrar a potencialidade das ferramentas de layout do software Erdas Imagine, realizou-se uma composição da imagem obtida pelo algoritmo (Máxima Verossimilhança) que apresentou o melhor resultado da classificação. Essa imagem pode ser observada na Figura 9. 
AVALIAÇÃO DE ALGORITMOS DE CLASSIFICAÇÃO SUPERVISIONADA DE IMAGEM DIGITAL DO SATÉLITE IKONOS NA REGIÃO DA SERRA DO SALITRE - MG

Imagem Temática da Região da Serra do Salitre - MG

(4)

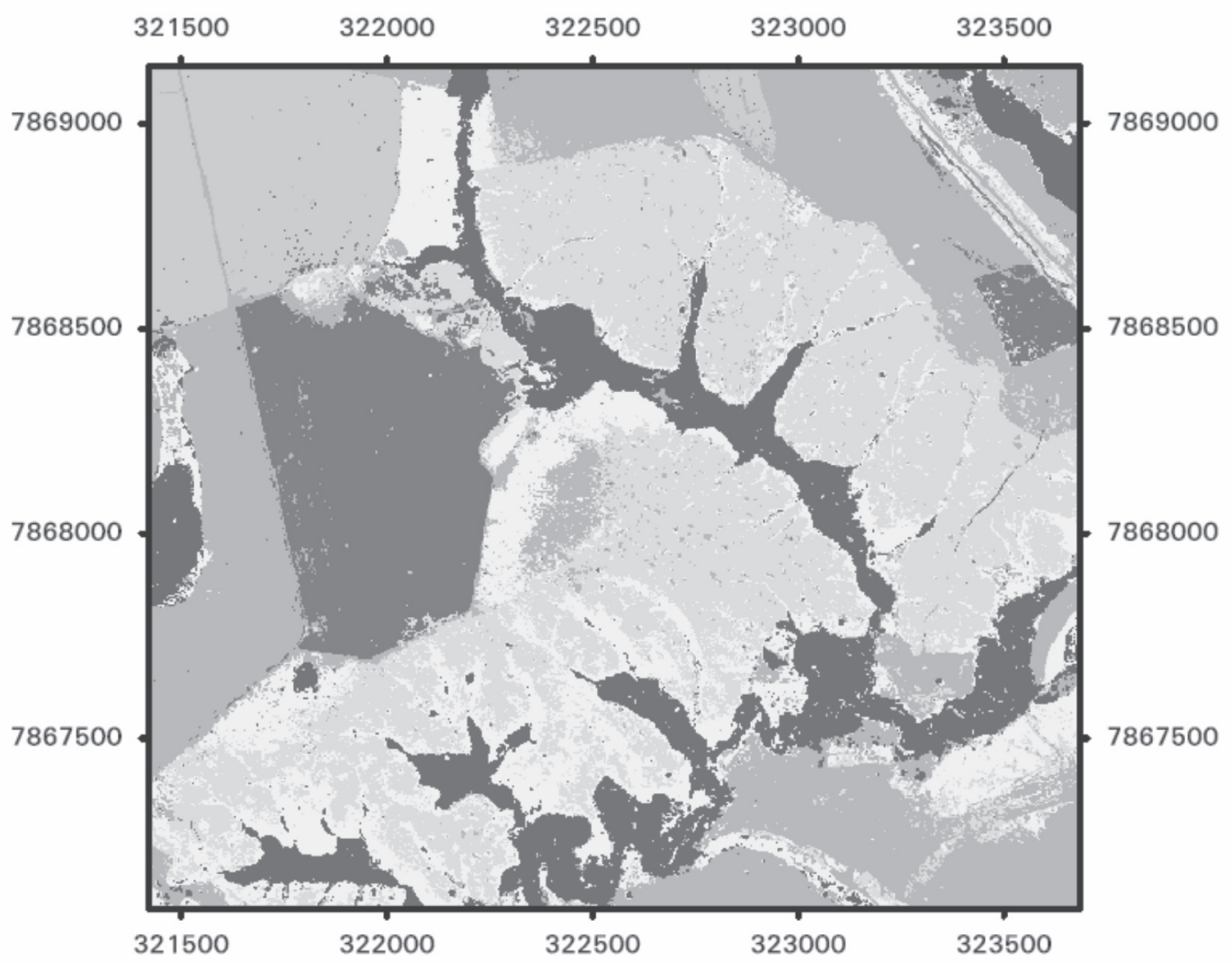

321500

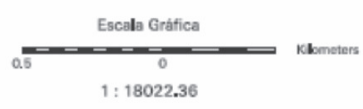

Classes Temáticas

1: 18022.36

Class_Names

campo limpo

campo sujo

café

braquiária

mata

pasto

solo

Figura 9. Imagem temática classificada obtida pelo algoritmo MAXVER. 


\section{CONCLUSÃO}

A análise dos resultados permite concluir que, considerando-se as imagens fornecidas (variáveis discriminatórias) e as características da área em estudo, o pior desempenho dentre os classificadores foi verificado pelo classificador da Distância Mínima até a Média e os melhores resultados de desempenho na classificação foram observados pelo classificador da Máxima Verossimilhança.

Pode-se concluir ainda que tanto a coleta das amostras de treinamento como as técnicas de avaliação de qualidade dessas amostras desempenharam um papel importante para o resultado final, entretanto, a escolha de uma adequada regra de decisão (algoritmo ou método de classificação) é fundamental para se obter um resultado satisfatório.

\section{REFERÊNCIAS BIBLIOGRÁFICAS}

Crósta, A. P. Processamento digital de imagens de sensoriamento remoto, Unicamp, Gráfica Universitária 170p., 1993

Fonseca, L. M. G. Processamento digital de imagens, Instituto Nacional de Pesquisas Espaciais - INPE. 2000.

Mather, P. M. Computer Processing of Remotely-Sensed Images: An Introduction. Second Edition, Chichester: John Wiley and Sons. 292p., 1999.

Soares, V. P. Material de aula de sensoriamento remoto, Notas de aula ENF610. Universidade Federal de Viçosa UFV. 73p., 2000. 